\title{
Comparative studies on the corneal structural adaptation of two rodents inhabiting different environments
}

\author{
El-Sayed Fikri Ali El-Dawi ${ }^{1}$ and Hamdy Aly ${ }^{2}$ \\ 1- Department of Zoology, Faculty of Science, Ain Shams University, Cairo, Egypt. \\ 2- Department of Anatomy, Faculty of Medicine, Ain Shams University, Cairo, Egypt.
}

\begin{abstract}
The corneas of Rattus rattus and Meriones shawi are composed of three main layers; an outer epithelium, a middle stroma (basement membrane, total stroma and Descemet's membrane) and an inner endothelium. The mean thickness of the epithelium, total stroma, Descemet's membrane, and endothelium was about $52 \pm 7.3 \mu \mathrm{m}, 275 \pm 18.7 \mu \mathrm{m}, 5.5 \pm 0.7 \mu \mathrm{m}$, and $7.5 \pm 0.23 \mu \mathrm{m}$ in $R$. rattus, whereas it was $38 \pm 5.8 \mu \mathrm{m}, 124 \pm 4.7 \mu \mathrm{m}, 4 \pm 0.21 \mu \mathrm{m}$, and $4.2 \pm 0.17 \mu \mathrm{m}$ in $M$. shawi.

In $R$. rattus and $M$. shawi, the outermost cells of the corneal epithelium are mostly polygonal and hexagonal in shape with nearly regular borders and show a dense pattern of microplicae with different scatter electron that exhibits three and two polymorphic appearances, respectively. Type A: numerous light cells with dense microplicae; type B: many dark cells with a moderate density of microplicae, and type $\mathrm{C}$ : few dark cells with a less density of microplicae are found in $R$. rattus; whereas, types $\mathrm{A}$ and $\mathrm{B}$ are found in $M$. shawi.

In both investigated species, the epithelial cells are characterized by some structural components, such as glycocalyx, fibrous components and tight junction between these cells, to resist the impact of the external stressed factors and to protect the underlying tissue, as well as to maintain an excellent transparency of the cornea. Among these structures, the cytokeratin filaments are the major components of the cytoplasm of the corneal epithelial cells (basal, polygonal, wing and squamous cells). Actin filaments are also found in the corneal epithelial cells, but they are prominent within the apical epithelial cells.

In $R$. rattus, the stroma is formed of an outer lamellar zone and an inner lamellar one; the latter is thicker and characterized by its interfibrillar spaces between the bundles of wavy dissociated collagen fibrils, which are arranged in an orthogonal manner. In M. shawi, however, the stroma is formed of one lamellar zone of flattened bundles of highly wavy and branched collagen fibrils, which are composed of perpendicular fibrillae alternating with longitudinal ones.

In $R$. rattus, the SEM showed that the endothelial cell surfaces are slightly bulging with many blebs, whereas in $M$. shawi, it showed that the surfaces of the endothelial cells are flattened and nearly smooth.

In conclusion, the transparency of the cornea, may be highly attributed to the increase in the thickness of the stroma, the presence of stromal interfibrillar spaces and the case of the stromal swelling. These aforementioned features are found in the corneal stroma of $R$. rattus, which live in different habitats of varying degrees of density such as water and dry or humid air, whereas these features are lacking in $M$. shawi, which live only in arid zones.
\end{abstract}

Key words : Cornea , mammals, rodents, adaptation, environment, LM, SEM, TEM

\section{Introduction}

The transparent nature of the cornea and its importance in the visual pathway as a major refracting lens of the eye have intrigued the investigators in many different disciplines, and their studies have added immeasurable knowledge to the understanding of the cornea in health and disease of human (Svedbergh and Bill, 1972; 
Smolin and Thoft, 1994; Beuerman and Pedrosa,1996; Krachmer et al.,1997) and other mammals such as the experi-mental rodents (Lois et al., 2003; Takami et al., 2004). Other investigations were applied on the corneal structure of the domesticated and experimental mammals, such as dogs and pigs (Collin and Collin, 1998; Svaldeniene et al., 2003), rabbits (Hodson et al., 1977; Doughty, 1996; Collin and Collin, 1998; Doughty et al., 2001; Doughty and Bergmanson, 2004) and albino rats and mice (Amemiya, et al., 1980; Haustein, 1983; Yamaguchi et al., 1992; Collin and Collin, 1998 ; Sasaki et al., 1999). Most of the aforementioned studies dealt with certain corneal layers, such as the epithelium (Amemiya, et al.,1980; Doughty , 1996; Takami et al., 2004), the stroma (Haustein, 1983; Doughty et al., 2001; Doughty and Bergmanson, 2004) and the endothelium (Hodson et al., 1977; Yamaguchi et al.,1992 ; Collin and Collin, 1998 ; Sasaki et al., 1999 ).

Except the investigations on the corneal epithelium of the false killer whale and the Australian marsupial koala (Collin and Collin, 2000), however, no attempts, as far as known, have been made to study the corneal structure of the other wild small mammals. Among these wild mammals, the rodents are considered the most widely distributed mammals all over the world and adapted to various habitats (desert, rocky, sandy, vegetative, tunnels....etc) of different climatic factors (temperature, light...etc). Moreover, many of them are nocturnal and others are diurnal.

In accordance, the aim of this investigation is planned to study the corneal structural adaptation, as a comparative study, of two wild species of rodents, the black rat, Rattus rattus, and the shaw's jird, Meriones shawi, which live in two different habitats; the first species inhabits the vegetative and civilized areas of the Nile Valley, countries, houses, tunnels, stores... etc, whereas the second species inhabits the rocky desert. With the aid of light and electron microscopy (scanning and transmission), high hopes that this study may provide us a fair knowledge about the adaptive structure of the cornea in these habitats and also may represent a preliminary investigation for our further studies on other mammalian species.

\section{Materials And Methods}

Specimen collection and dissection:

Ten adult species of both the black rat, Rattus rattus (Family : Muridae), and the shaw's jird, Meriones Shawi (Family: Cricetidae), were used in this study. Rattus rattus were collected from Abou Rawash at Giza, whereas M. Shawi were collected from Borg Al-Arab near Alexandria. The average lengths for $R$. rattus and $M$. shawi were as follows: for the head and body, they were $165,4 \mathrm{~mm}$, and $140,2 \mathrm{~mm}$, whereas for the tail, they were $205,3 \mathrm{~mm}$ and $135,7 \mathrm{~mm}$, respectively.

The head of each species studied, was sacrificed and both eyes were carefully and immediately enucleated and cleansed of extraneous tissues with fine forceps and iris scissors. The eyes were then hemisected with a razor blade and the central corneal cups were taken out under a dissecting microscope and prepared for light, transmission and scanning electron microscopy.

\section{Light and transmission electron microscopy (LM \& TEM):}

The dissected central corneas were cut into small pieces and fixed in cold $\left(4^{\circ} \mathrm{C}\right) 2.5$ $\%$ glutraldehyde in $0.2 \mathrm{M}$ phosphate buffer (pH 7.4) for 2 hours and post-fixed (2 hours at $4^{\circ} \mathrm{C}$ ) in $\mathrm{OsO}_{4}$ for electron microscopy. The samples were then dehydrated, treated with propylene oxide, infiltrated and embedded in Epon 812. Semithin sections were cut with the RMC-MT7 ultramicrotome and stained with toluidine blue. The corneas were then examined and photographed by Olympus light microscope (LM) and the measurements of their different layers were taken using the eye piece and slide micrometers.

Ultrathin sections (silvery) were cut, using a diamond knife and stained with uranyl acetate and lead citrate. The sections were then examined and photographed using a Joel 1010 Transmission Electron Microscope at the Regional Center for Mycology and Biotechnology, Al-Azhar University.

\section{Scanning electron microscopy (SEM):}

The dissected corneas were cut into small pieces and fixed in $2 \%$ glutral- 
dehyde- $2 \%$ paraformaldehyde in $0.1 \mathrm{M}$ phosphate buffer, $\mathrm{pH} 7.4$ at room temperature for 60 minutes. They were then washed twice in buffered sucrose $(0.1 \mathrm{M}$ phosphate buffer, 5\% sucrose solution) for 5 minutes. The tissues were post-fixed in $2 \%$ osmium tetraoxide $\left(\mathrm{OsO}_{4}\right)$ with $2 \%$ phosphate buffer for 2 hours at $4^{\circ} \mathrm{C}$. After fixation, the tissues were dehydrated in upgraded series of ethanol. They were further dehydrated in absolute ethanol : acetone (1:1) solution for further 30 minutes, then in absolute acetone for 3 - 10 minutes. The tissues were dried in $\mathrm{CO}_{2}$ drying apparatus (Critical-Point Dryer, CPD 030) and mounted on stubs, then coated with gold in Sputter Coater (SCD 005). They were examined and photographed using a Philips Scanning Electron Microscope XL30 at the Department of Anatomy, Faculty of Medicine, Ain Shams University.

\section{Statistical Analyses:}

The standard deviation, statistical analyses and significant levels $(P)$ for all measurements were made by using Student's $t$-test.

\section{Results}

The mean thickness of the total corneas of both Rattus rattus and Meriones shawi measures about $334.5 \pm 21.9 \mu \mathrm{m}$ and $166.2 \pm 14.5 \mu \mathrm{m}$, respectively and table (1), shows a highly significantly difference $(p<0.001)$ of them.

\section{Light microscopy:}

The corneas of both species are composed of three main layers; an outer epithelium, a middle stroma (basement membrane, total stroma and Descemet's membrane) and an inner endothelium (Figs.1-6). The mean thickness of the epithelium, total stroma, Descemet's membrane, and endothelium is about $52 \pm$ $7.3 \mu \mathrm{m}, 275 \pm 18.7 \mu \mathrm{m}, 5.5 \pm 0.7 \mu \mathrm{m}$ and $7.5 \pm 0.23 \mu \mathrm{m}$ in $R$. rattus, whereas it is 38 $\pm 5.8 \mu \mathrm{m}, 124 \pm 4.7 \mu \mathrm{m}, 4 \pm 0.21 \mu \mathrm{m}$ and $4.2 \pm 0.17 \mu \mathrm{m}$ in $M$. shawi.

Table (1), shows that, in $R$. rattus and $M$. shawi, there are very highly significantly differences $(P<0.0001)$ in the mean thickness of both the total stroma and endothelium, a highly significantly difference $(P<0.001)$ in the mean thickness of the
Descemet's membrane, and a significantly difference $(P<0.05)$ in the mean thickness of the epithelium.

Epithelium: The epithelial layer of $R$. rattus and $M$. shawi overlies a thin and distinct stromal basement membrane; it is composed of a stratified squamous, nonkeratinized epithelium (about 9 rows of cells). These cells are arranged as a basal monolayer of short columnar cells, an intermediate polygonal cells (about 4 cells thick), an outer layer of flattened polygonal cells (wing cells) of about 3 cells thick and an outermost layer formed of flattened squamous cells (one cell thick) (Figs.2 \& 5). It must be noted that some dark epithelial cells have been observed in between the epithelial cells near the basal layer (Figs. $2 \& 5$ ).

Stroma: In $R$. rattus, it consists of an outer thin (about $72 \pm 12.5 \mu \mathrm{m}$ ) lamellar zone of less distinguishable parallel collagen fibrils, and an inner thick (about $203 \pm 17.6 \mu \mathrm{m}$ ) lamellar zone of dissociated ones by many large interfibrillar spaces (Figs. 2 \& 3). However, in M. shawi, it is composed of distinct and compact collagen lamellar fibrils, which are regularly arranged as parallel fibrils to each other in a wavy and branched manner (Figs. $5 \& 6$ ).

In $R$. rattus, few elongated keratocytes are observed scattering in the whole stroma (the outer and inner lamellar zones), however, in M. shawi, these cells are more distinguishable, great in number and scatter in between the lamellae of the stroma (Figs.2,3,5 \& 6). The stroma of both species lies above a moderately thick and distinct Descemet's membrane (Figs. 3 \& 6).

Endothelium: In $R$. rattus and $M$. shawi, the endothelium is the innermost layer bordering the anterior chamber of the eye and is formed of a single layer of illdefined low cuboidal cells underlying the Descemet's membrane (Figs. $3 \& 6$ ).

\section{Scanning and transmission electron microscopy:}

Epithelium: In R. rattus and M. shawi, the SEM shows that the outermost cells of the corneal epithelium are mostly polygonal and hexagonal in shape with nearly regular borders and show a dense pattern of microplicae with different scatter electron that exhibits three and two polymorphic 
appearances, respectively. Type A: numerous light cells with dense microplicae; type B: many dark cells with a moderate density of microplicae, and type C: few dark cells with a less density of microplicae are found in $R$. rattus; whereas, types $\mathrm{A}$ and $\mathrm{B}$ only are found in $M$. shawi (Figs.7\& 8). It must be noted that minute microholes have been observed on the surfaces of the apical epithelial cells of $R$. rattus (Fig.9), as well as on those of $M$. shawi.

The TEM shows that, in both investigated species, the outermost apical cells possess numerous and minute microplicae, which are coated with extensive glycocalyx to the extent that the microplicae can not be easily seen. Moreover, the so termed wing cells of the outer layer possess lateral thin wing-like extensions from the main bulks of the cell bodies (Figs.10 a \& b). The basal cell layer appeared to be the most active mitotically epithelial cells (Fig.11). It must be noted that the major components of the cytoplasm of the corneal epithelial cells (basal, polygonal, wing and squamous cells), are the cytokeratin filaments (Figs.12\&13). Actin filaments are also found in the corneal epithelial cells, but they are prominent within the apical epithelial cells (Figs.10a \& b). Moreover, microtubules are also present in the corneal epithelium, but they are prominent within the mitotic basal cells (Figs.12 \&13). Besides, the corneal epithelial cells possess a sparse accumulation of mitochondria, Golgi bodies, endoplasmic reticulum and small vesicles.

The membranes of the epithelial cells are highly interdigitated. Desmosomes are prominent cell-to-cell adhesion junctions along the cell borders. Gap junctions are also found between the lateral membranes of the wing cells (Figs. $10 \mathrm{a} \& \mathrm{~b}$ ). The basal cells of the epithelium adhere to their basement membrane and the underlying fibrils of the stroma through an adhesion complex. A foam layer of newly synthesized collagen fibrils appeared in the interface of the epithelium and the upper part of the stroma (Fig.13). In $R$. rattus and M. shawi, no Bowman's layer was observed under the basement membrane.

Stroma: The stromal layers, of both investigated species, are nearly similar in their general arrangement, but differ in their thickness and composition.

In $R$. rattus, the stroma is formed of outer and inner lamellar zones which differ in their thickness (Table 1). The outer lamellar zone occupies about the anterior third of the stroma and has wavy and branched collagen fibrils. On the other hand, the inner lamellar zone occupies the main posterior part of the stroma and is characterized by its interfibrillar spaces between the more wavy and dissociated bundles of collagen fibrils, which are arranged in an orthogonal manner i.e. at right angle to another (Figs. 13 and $14 \mathrm{a} \& \mathrm{~b}$ ).

In $M$. shawi, the stroma is formed of one lamellar zone. It is composed of flattened bundles of highly wavy and branched collagen fibrils, especially those near the epithelium. The lamellar stroma is composed of perpendicular fibrillae alternating with longitudinal ones (Figs. 5, 6, 15 \&16).

The lamellar stroma, of both studied species, is secreted and maintained by the keratocytes, which are resided between the fibrils and each possesses a large elongated nucleus, Golgi bodies and endoplasmic reticulum. These keratocytes are great in number and size in $M$. shawi than those of $R$. rattus, while they are highly vesiculated in $R$. rattus, than those of $M$. shawi, which possess prominent granular endoplasmic reticulum (Figs.14a \& 16).

The Descemet's membrane, of both investigated species, is a regular, dark and exaggerated layer, which is synthesized by the endothelium and serves as a substratum for the endothelial cells. It is largely formed of parallel and very fine collagen fibrils (Figs.14b \& 15).

Endothelium: In $R$. rattus and $M$. shawi, the corneal endothelium is a single layer of low cuboidal cells, which appear by the SEM as irregular and flattened polygonal cells. In $R$. rattus, the surfaces of the endothelial cells appear slightly bulging with many blebs, however in M. shawi, their surfaces appear more flat and nearly smooth (Figs.17 \& 18). In both investigated species, the endothelial cells have large and flattened nuclei, a number of mitochondria, endoplasmic reticula and Golgi bodies. In $R$. rattus, the endothelial cells are characterized by the presence of a small 
number but prominent large vacuoles, as well as great numbers of vesiculated structures of the granular endoplasmic reticulum, however in $M$. shawi, the cytoplasm of the endothelial cells is highly granulated with some scattered vesiculated structures of the endoplasmic reticulum (Figs. $19 \& 20$ ).

Table 1: The mean thickness of the different corneal layers of the black rat, Rattus rattus, and the shaw's jird, Meriones shawi.

\begin{tabular}{||c||c|c|c||}
\hline $\begin{array}{c}\text { Corneal } \\
\text { layers }\end{array}$ & $\begin{array}{c}\text { Rattus rattus } \\
\text { Mean thickness } \\
(\mu \mathrm{m}) \pm \mathrm{SD}\end{array}$ & $\begin{array}{c}\text { Meriones shawi } \\
\text { Mean thickness } \\
(\mu \mathrm{m}) \pm \mathrm{SD}\end{array}$ & $P$ \\
\hline \hline Total cornea & $334.5 \pm 21.9$ & $166.2 \pm 14.5$ & $<0.001$ \\
\hline \hline Epithelium & $52 \pm 7.3$ & $38 \pm 5.8$ & $<0.05$ \\
\hline \hline Total stroma & $275 \pm 18.7$ & $124 \pm 4.7$ & $<0.0001$ \\
\hline \hline O L Z & $72 \pm 12.5$ & - & - \\
\hline \hline I L Z & $203 \pm 17.6$ & $4 \pm 0.21$ & $<0.001$ \\
\hline \hline $\begin{array}{l}\text { Descemet's } \\
\text { membrane }\end{array}$ & $5.5 \pm 0.7$ & $4.2 \pm 0.17$ & $<0.0001$ \\
\hline \hline Endothelium & $7.5 \pm 0.23$ & - & \\
\hline
\end{tabular}

N.B. $\quad P<0.05 \quad$ (significant)

$P<0.001 \quad$ (highly significant)

$P<0.0001 \quad$ (very highly significant)

$\mathrm{O} \mathrm{L} Z=$ Outer lamellar zone of the stroma

I $\mathrm{L} \mathrm{Z}=$ Inner lamellar zone of the stroma

\section{Explanation Of Figures}

Figs. 1 - $3 \& 4$ - 6: Photomicrographs of vertical semithin sections of the corneas of Rattus rattus, and Meriones shawi, respectively, showing the general structure of the cornea (the epithelium: Ep; basement membrane: B; stroma: S; keratocytes: K; Desemet's membrane: $\mathrm{D}$; and endothelium: En). Toluidine blue.

Figs.1 \& 4: Show the structure of the cornea and the thickness of its different layers. Notice the interfibrillar spaces (asterisks) and the outer and inner lamellar zones (OLZ and I LZ) in figure 1.(X 138).

Figs.2 \& 5: Show the structure of the epithelial layer (Ep), the dark cells (arrows), the wing cells (asterisks), the basement membrane (B) and the underlying stroma $(\mathrm{S})$. Notice also the differences between the stroma of both species, the keratocytes (K), and the branched collagen fibrils in figure 5 (white arrows).

( X 435).

Figs.3 \& 6: Show the structure of the stroma (S), the distinct Descemet's membrane (D) and the endothelium (En).

Notice many and large interfibrillar spaces (asterisks) in between the lame- llae of the inner lamellar zone (ILZ) and the keratocytes $(\mathrm{K})$ of the stroma of $R$. rattus (Fig.3). Also notice the distinct lamellar stroma with distinct keratocytes (K) of M. shawi (Fig. 6). ( $\mathrm{X}$ 435).

Figs. 7 \& 8: Scanning electron micrographs of the corneas of $R$. rattus and $M$. shawi, respectively, showing the different types of the apical epithelial cells.

Fig.7 : Shows type A, B and C cells. Notice the nearly regular borders of the cells.

(Scale bar $=20 \mu \mathrm{m}$ )

Fig.8 : Shows type A and B. Notice the slightly regular borders of the cells.

$($ Scale bar $=10 \mu \mathrm{m})$

Fig. 9 : Scanning electron micrograph of the cornea of $R$. rattus, showing the microholes (arrows) on the surfaces of the apical epithelial cells

$$
(\text { Scale bar }=2 \mu \mathrm{m})
$$

Figs.10a \&b: Transmission electron micrographs of the corneas of $R$. rattus and $M$. shawi, respectively, showing the structure of the epithelial cells.

Fig. 10a : Notice the surface of the apical cell is coated with extensive glycocalyx (arrows). Notice also the wing cells (asterisks), the tight adhesion between 
the epithelial cells (short arrows) and the gab junctions (arrowheads).

$$
(\text { Scale bar }=2 \mu \mathrm{m})
$$

Fig. 10b : Notice the microplicae (Mp), extensive glycocalyx (arrows) and exten-sive dark fibrous filamentous structures (white arrows) in the outer cells. Notice also the wing cells (star) and the tight adhesion (short arrows).

$($ Scale bar $=2 \mu \mathrm{m})$

Fig.11 : Transmission electron micrograph of the cornea of $R$. rattus, showing the structure of the epithelial cells. Notice the mitotically active nuclei $(\mathrm{N})$ of the basal cells that rest on a very thin basement membrane (B) and overlying less distinguishable fibrils of the outer lamellar zone (OLZ) of the stroma (S). Notice also the dark cells (asterisks) and many cytop-lasmic vacuoles (V).

$$
(\text { Scale bar }=10 \mu \mathrm{m})
$$

Fig. 12 : Transmission electron micr-ograph of the cornea of M. shawi, showing the microtubules (arrows)in an epithelial cell. Notice also extensive fine cytokeratin filaments (short arrows).

$$
(\text { Scale bar }=500 \mathrm{~nm})
$$

Fig. 13 : Transmission electron micrograph of the cornea of $R$. rattus, showing the basal cells that rest on very thin basement membrane (B) and overlying less distinguishable fibrils of the outer lamellar zone (OLZ) of the stroma (S) and the foam appearance (star). Notice the microtubules (arrows), fine cytokeratin filaments (short arrows). Notice also the adhesion complex (white arrows). $\quad$ (Scale bar $=2 \mu \mathrm{m}$ ).

Figs.14a\&b: Transmission electron micrographs of the cornea of $R$. rattus, showing the structure of the lamellar stroma.

Fig.14a: Shows the orthogonal arrangement of the collagen fibrils (arrows) of the inner lamellar zone (ILZ) and an elongated keratocyte $(\mathrm{K})$ with extensive vacuoles $(\mathrm{V})$ and a large nucleus $(\mathrm{N})$. Notice the interfibrillar spaces (asterisks). $\quad$ (Scale bar $=10 \mu \mathrm{m})$.

Fig.14 b: Shows the wavy arrangement of the collagen fibrils of the inner lamellar zone (ILZ) and the interfibrillar spaces (asterisks). Notice the structure and relation of the Descemet's membrane (D) with the inner lamellar zone (ILZ) and the endothelium (En) which possess large vacuoles (V).

(Scale bar $=10 \mu \mathrm{m})$.

Figs. 15 \& 16: Transmission electron micrograph of the cornea of M. shawi, sho-wing the structure of the lamellar stroma.

Fig. 15 : Shows the wavy appearance of the lamellar stroma (S) and an elongated keratocyte $(\mathrm{K})$, with a large nucleus $(\mathrm{N})$. Notice the structure and relation of the Descemet's membrane (D) with the stroma and endothelium (En).

(Scale bar $=2 \mu \mathrm{m})$.

Fig. 16: Shows the arrangement of the perpendicular (dark star) and longitudinal (white star) collagen fibrillae. Notice the structure of the keratocyte (K) with its prominent granular endoplasmic reticulum (ER).

(Scale bar $=500 \mathrm{~nm}$ ).

Figs.17 \& 18 : Scanning electron micrographs of the corneas of $R$. rattus and $M$. shawi, respectively, showing the irregular polygonal endothelial cells, and the nature of their surfaces.

Fig. 17: Shows the irregular cell borders and the slightly bulging surfaces. Notice the small blebs on the surfaces.

$($ Scale bar $=20 \mu \mathrm{m})$.

Fig. 18: Shows the irregular cell borders and the smooth surfaces of the endothelial cells. (Scale bar $=20 \mu \mathrm{m}$ ).

Figs.19\&20: Transmission electron micrographs of the corneas of $R$. rattus and $M$. shawi, respectively, showing the structure of the endothelium (En)and its relation to the Descemet's membrane (D).

Fig. 19 : Shows the highly vesiculated structure of the endothelial cells. Notice a large vacuole (asterisk) and the nucleus $(\mathrm{N})$. $\quad($ Scale bar $=2 \mu \mathrm{m})$.

Fig.20: Shows the highly granulated cytoplasm of the endothelial cells, scattered vacuoles $(\mathrm{V})$ and mitochondria $(\mathrm{M})$.

$($ Scale bar $=2 \mu \mathrm{m})$. 
El-Sayed Fikri Ali El-Dawi \& Hamdy Aly
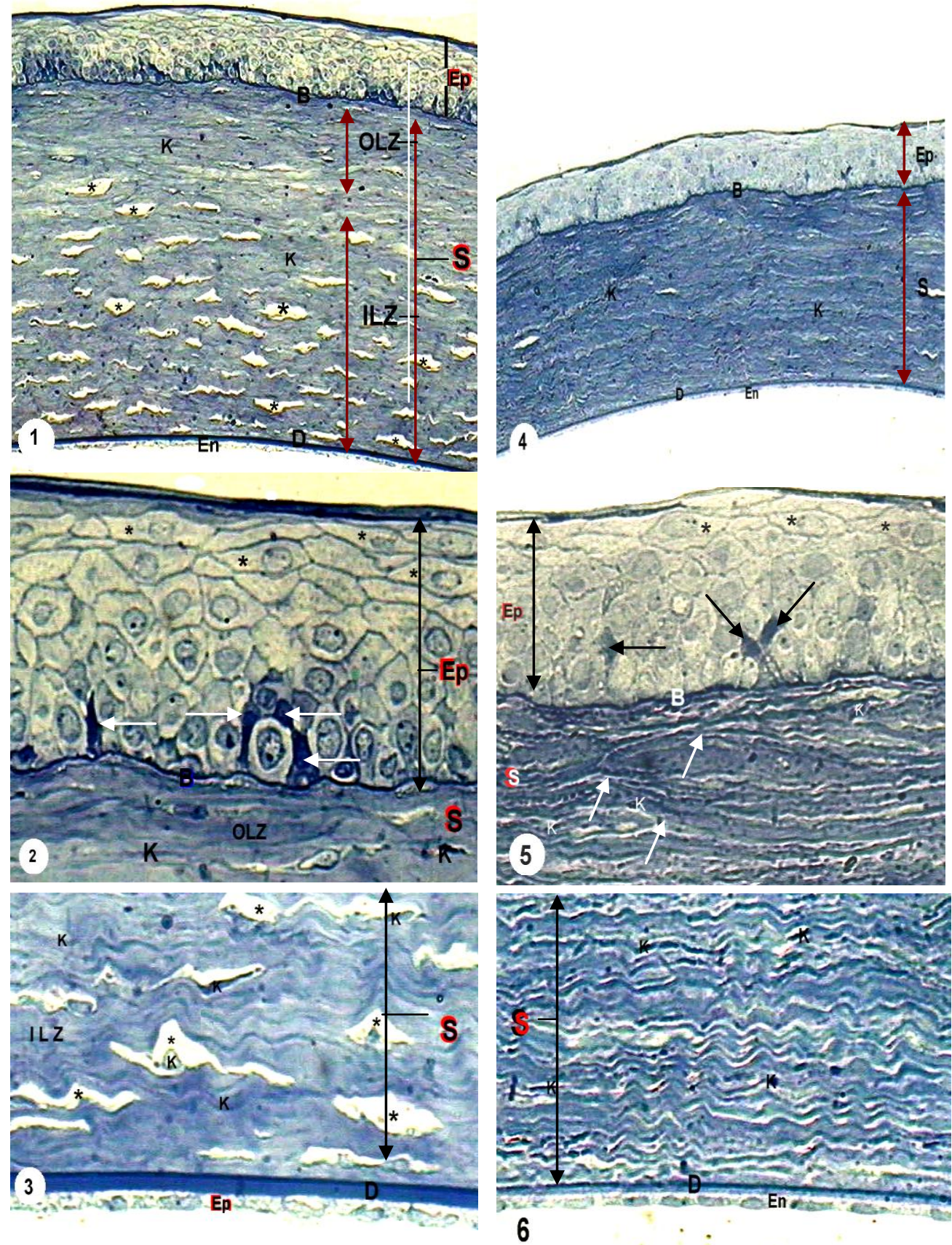
Comparative studies on the corneal structural..
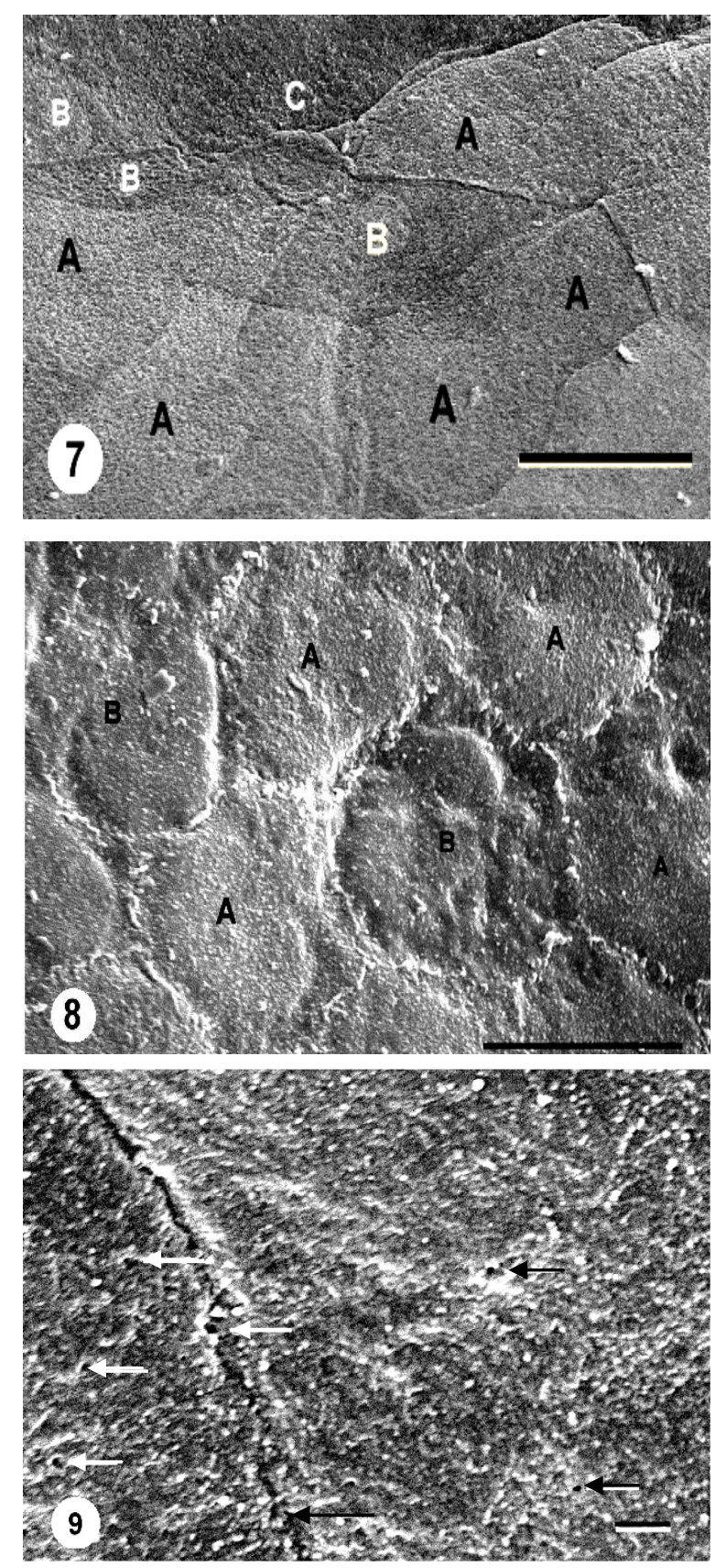

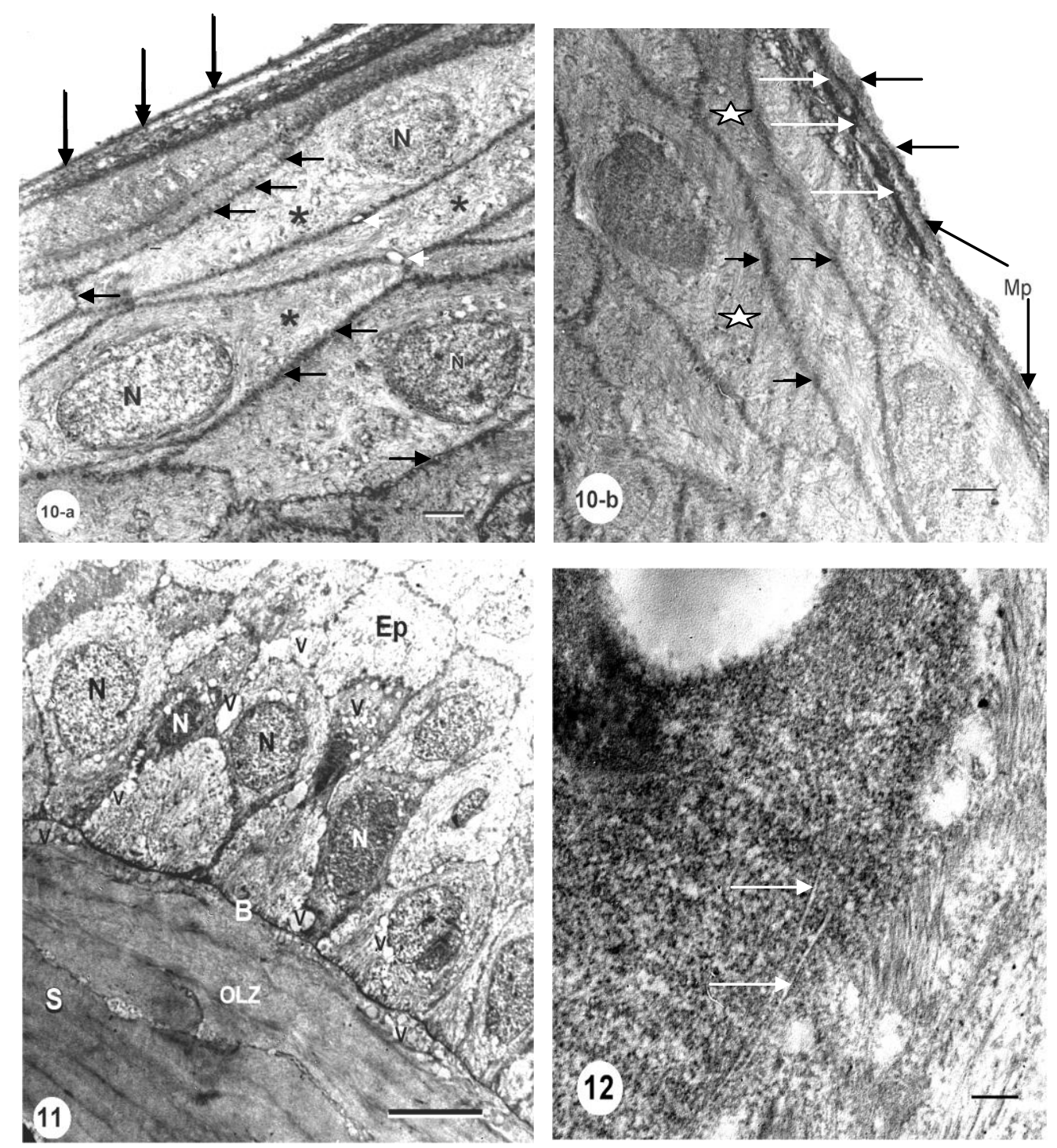

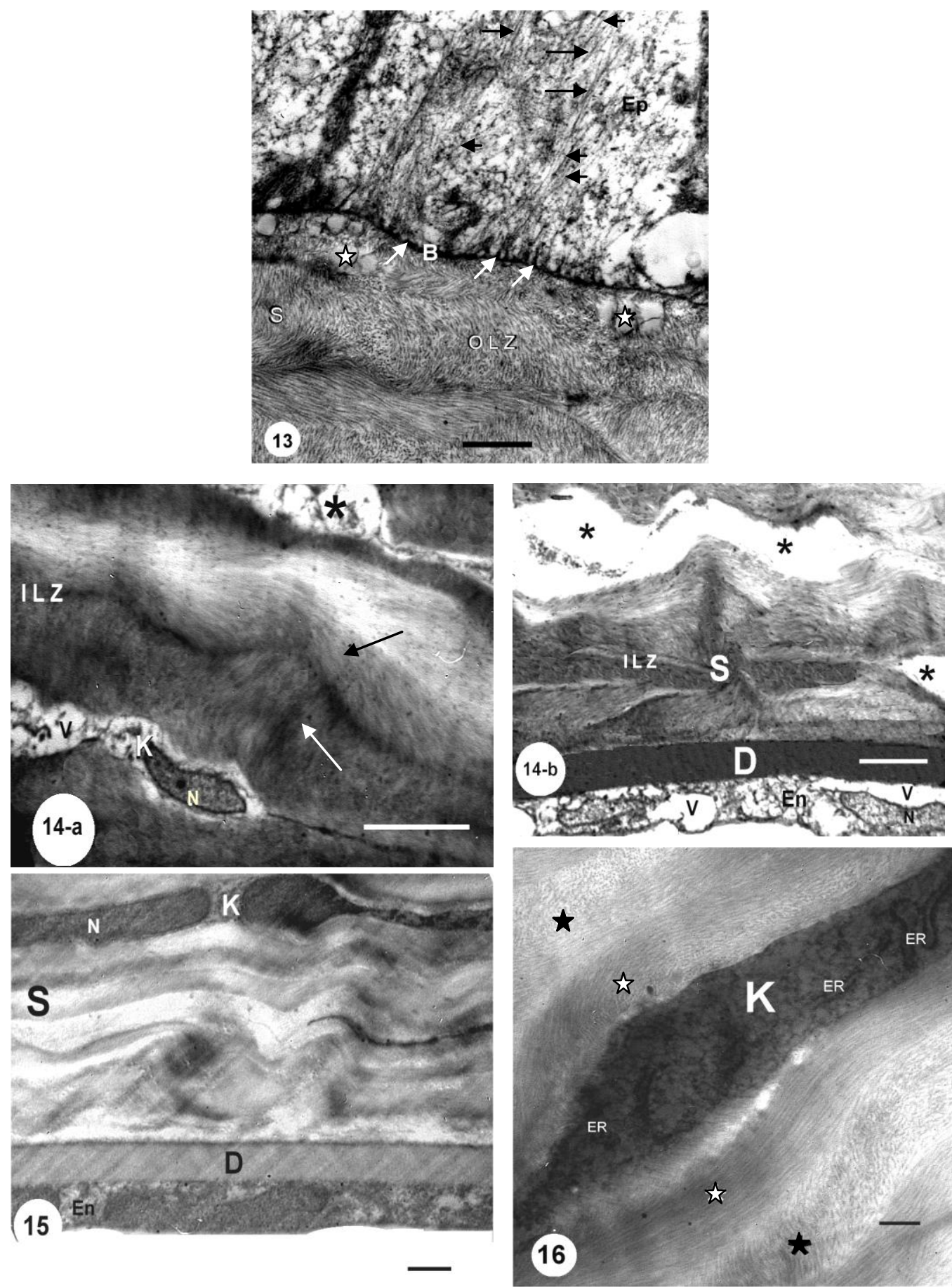


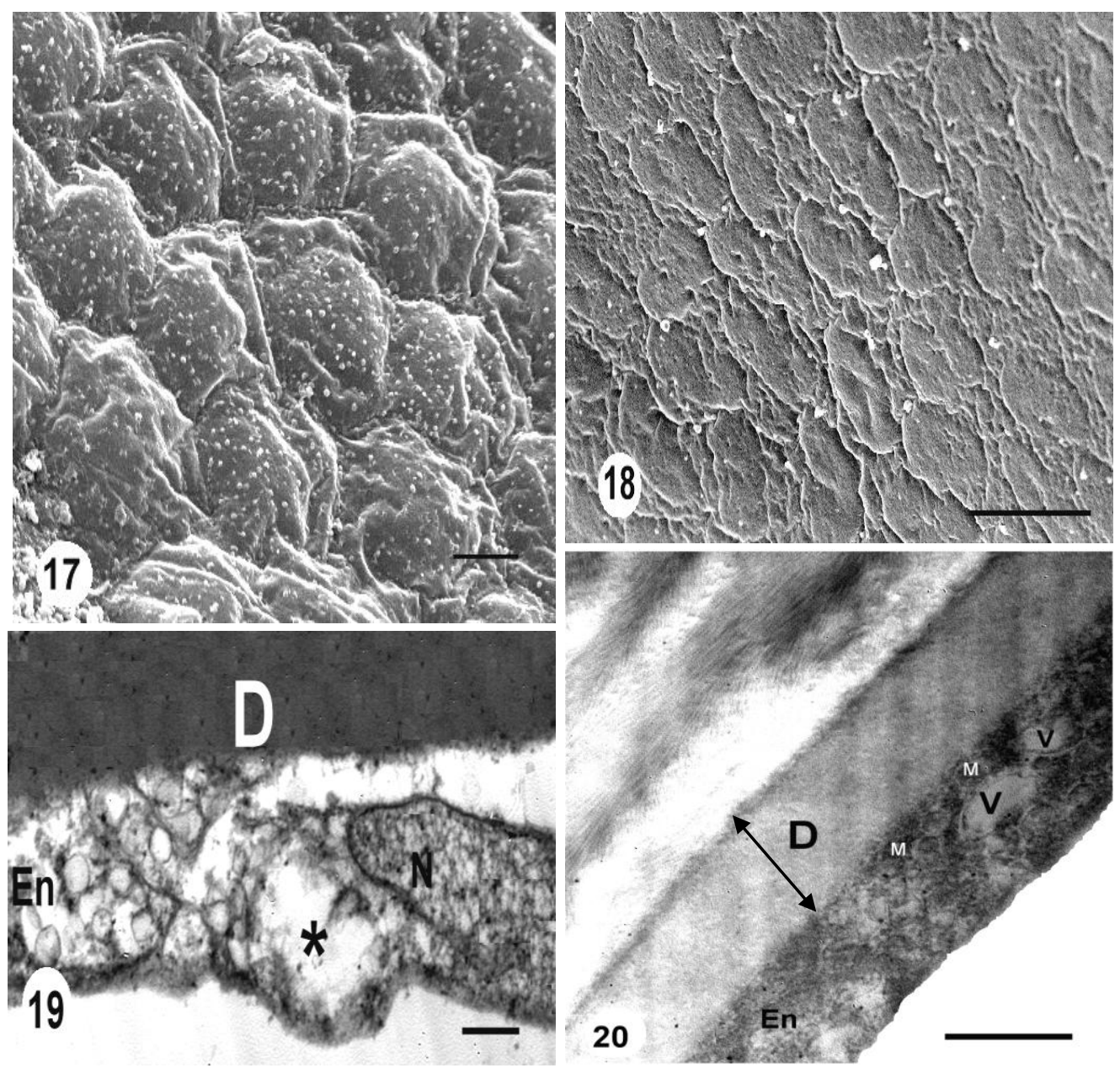




\section{Discussion}

The black rat, Rattus rattus, and the shaw's jird, Meriones shawi, are two rodents living in two different habitats; the first species inhabits the Nile Valley, countries, houses, tunnels, stores...etc, whereas the second species inhabits the rocky desert (Wassif, 1995). These habitats have characteristic climatic factors affecting the structure of the different organs of their animals. Temperature is considered as one of the main factors affecting the structure of the skin and cornea. Consequently, the cornea of the desert animals (M. shawi) is subjected to a great effect of the high temperature rather than that of $R$. rattus inhabiting the Nile Valley of the moderate temperature. In spite of these two species are related to order Rodentia and suborder Myomorpha, they are apparently have some morphological and structural differences of their corneas according to the mode of life and type of habitat.

The general corneal structure of both, $R$. rattus and $M$. shawi, is nearly similar to each other, as it is composed mainly of three layers: the epithelium, stroma (basement membrane, total stroma and Descemet's membrane) and endothelium. Such general structure is somewhat similar to what has been described for the corneas of most vertebrate classes including human and other mammals (Smolin and Thoft, 1994; Beuerman and Pedrosa,1996; Krachmer et al., 1997; Chakravarti, 2001; El-Dawi, 2002 \& 2004; Svaldeniene, et al., 2003).

\section{Epithelium}

The epithelial layer of $R$. rattus and $M$. shawi is composed of a stratified squamous, non-keratinized epithelium (about 9 rows of cells), which are arranged as a basal monolayer of short columnar cells, an intermediate polygonal cells (about 4 cells thick), an outer layer of wing cells (about 3 cells thick), and an outermost layer of flattened squamous cells (one cell thick). Similar arrangement and number have been described in the knockout mice (Chakravarti, 2001). However, Gibson
(1994) stated that the mammalian corneal epithelium is composed of 5 - 7 cell layers arranged as basal columnar cells, one to three cell layers of intermediate wing cells and an outer cell layers of three to four flattened squamous cells. Also, Krachmer et al. (1997) described 5 - 6 layers of three corneal epithelial cell types of mammals including human; these types were monolayer basal columnar cells, two to three layers of wing cells and two to three layers of superficial cells. In the present investigation, the increase in the number of the epithelial layers and types of cells, most probably, for protection the underlying stroma from the extreme external factors which are highly correlated to the variable and hard habitats in which they live.

In $R$. rattus and $M$. shawi, the SEM shows that the outermost corneal epithelial cells are mostly polygonal and hexagonal in shape with nearly regular borders. They possess microholes and show a dense pattern of microriplicae. Such appearance simulates that found in human and most studied mammals (Gibson, 1994; Doughty, 1996; Krachmer et al., 1997; Collin and Collin, 2000). In the rhino mouse (Amemiya, et al., 1980) and in the rat (Takami et al., 2004), the surfaces of the outermost corneal epithelial cells possess microvilli, microplicae and microholes which possess elevated rims formed by large folds of the apical epithelial cells. In human (Gibson, 1994) the apical epithelial cells possess numerous microplicae and microvilli, which are suggestive of the epithelium normal function.

In $R$. rattus and $M$. shawi, the dense pattern of microplicae shows different scatter electron that exhibits three and two polymorphic appearances, respectively; type A: light cells with dense microplicae; type B: dark cells with a moderate density of microplicae, and type C: dark cells with a less density of microplicae. In the white rabbit (Doughty, 1996) three cell types of the corneal epithelium (light, medium and dark), were distinguished. Also, Gibson (1994) demonstrated that the mammalian apical epithelial cells show three types of 
cells (light, moderate and dark) according to the degree of the scatter electron of the microplicae. However, two types of cells, dark and light were observed in the corneal epithelium of the piglets (young pigs). These two types not observed in young dogs and adult dogs and pigs (Svaldeniene, et al.,2003). Moreover, microvilli and microplicae have been observed on the rat corneal epithelium (Takami et al., 2004). On the other hand, in different mammals, Krachmer et al. (1997) distinguished two types of corneal epithelial cells, large dark cells and small light ones. The dark cells are covered with dense microvilli, whereas the light ones have fewer microvilli. Most probably, the presence of various types of the apical epithelial cells according to their microprocesses represents stages of developmental processes, as the light cells are the most recent, whereas the dark cells are the oldest ones. Moreover, these microprocesses, microplicae, microvilli or even microridges, most probably, are highly correlated to the external environments and their demands, as they enlarge the total cell surface area to allow the active exchange of oxygen and nutrients between the cells and tear fluid.

In the present investigation, the epithelial cells of both investigated species are characterized by some structural components, such as glycocalyx, fibrous compo-nents and tight junction between these cells, to resist the impact of the external stressed factors and to protect the underlying tissue, as well as to maintain an excellent transpar-ency of the cornea.

In $R$. rattus and $M$. shawi, the microplicae of the outermost apical cells were coated with extensive glycocalyx to the extent that they can not be easily seen. This is also observed in the guinea pigs and rats (Gibson, 1994; Svaldeniene, et al., 2003). Gibson (1994) described that the glycocalyx is loosely associated with the tear film layer, as well as with the mucin and tear film spreading over the surface of the eye. Moreover, Nishida (1997) stated that glycocalyx is structurally formed of floating particles of glycolipid and glycoprotein molecules in the cell mem-brane and covered by oligosaccharides. He suggested that this structure maintains the hydrophilic properties of the epithelial cells. Most probably, the presence of extensive glycocalyx, in the species of the present investigation, is apparently highly correlated to retain the tear fluid for long periods as possible for protection against microbial or fungal infections scattering in the different habitats of $R$. rattus or maintain the stability of the tear fluid under the stress of high temperature and preventing their eyes from drying as in the case of $M$. shawi which inhabits the rocky desert.

It $R$. rattus and $M$. shawi, the presence of the cytokeratin filaments in the cytoplasm of the corneal epithelial cells, as well as the actin filaments especially within the outermost epithelial cells was greatly similar to what have been reported in human and many mammalian species by Gibson (1994) and Nishida (1997). The latter author stated that the wing cells are rich in K45 keratins which are specific to non-keratinized corneal epithelial cells. In the knockout mice, Chakravarti (2001) stated that keratins (KI $-\mathrm{K} 20)$ form the intermediate filaments of the epithelial cells. Most probably, the extensive appearance of such fibrous components in the corneal epithelial cells of $R$. rattus and $M$. shawi, reflects their stiffness as a supportive cells against external stress. On the other hand, the highly interdigitation, the presence of extensive desmosomes and gap junctions between the corneal epithelium, especially the wing and intermediate cells, of the present investigation, have been also described in some mammalian species (Gibson, 1994; Smolin and Thoft, 1994; Nishida, 1997; Svaldeniene, et al., 2003). Most probably, the presence of these junctional complexes reflects the tight junctions of these cells. Such tight junctions have a mechanical barrier function and prevents the penetration of the exterior aqueous material easily to the corneal stroma, as in $R$. rattus that live in various habitats including the tunnels or from the stroma to exterior as in $M$. shawi that live in rocky desert of hot climate.

\section{Stroma}

The presence or absence of the Bowman's layer is still a matter of debate. 
In $R$. rattus and $M$. shawi, no Bowman's layer was observed under the basement membrane. It has been found that most species of mammals do not have the Bowman's layer (Gibson, 1994; Smolin and Thoft, 1994; Krachmer et al., 1997). However, Nishida (1997) stated that this layer is observed in human and certain other mammals including the primates. Moreover, Hayashi et al. (2002) and Svaldeniene, et al. ( 2003) found that the Bowman's layer is thick and well developed in higher mammals such as dogs, cattle and human; thin and ill-developed in the mouse, rat, guinea pig, rabbit and cat. The functional significance of the Bowman's layer remains a matter of discussion. Many authors suggested that it plays an important role in the maintenance of the epithelial structure, for maintaining epithelial uniformity, thus forming an appropriate refractive power or preventing the contact between the epithelium and stroma (Gibson, 1994; Smolin and Thoft, 1994; Krachmer et al., 1997; Nishida, 1997). In fact, the presence or absence of the Bowman's layer is greatly dependent on the epithelial structure in its ability to allow or prevent the passage of aqueous material to or from the stroma. This is apparently clear in $R$. rattus and $M$. shawi, who lack the Bowman's layer as their epithelial cells are formed of many layers and contain prominent cytokeratin and actin filaments, as well as the presence of tight junctions between these cells.

The transparency of the cornea is still subjected to different hypotheses. Nishida (1997) stated that the regular arrangement of the collagen fibrils, in mammals and human, contributes to the corneal transparency and any disturbance in the uniformity of the interfibrillar spaces cause loss of transparency. Freud et al, (1995) suggested that, in human and rabbit, the corneal transparency is the result of the small size of the constituent collagen fibrils, and the lamellae of the posterior stroma are more regular in arrangement than those of the anterior stroma. Most probably, the transparency of the cornea is highly attributed to the increase in the thickness of stroma, presence of interfibrillar spaces and the case of the stromal swelling. The aforementioned features are found in the corneal stroma of $R$. rattus, which live in different habitats of varying degrees of density such as water and dry or humid air as compared with $M$. shawi, which live only in arid zones and lack these features.

\section{Endothelium}

In $R$. rattus and $M$. shawi, the SEM showed that the corneal endothelial cells were flattened polygonal cells with irregular borders. In most vertebrates including human and other mammalian animals such as rabbits, rats, mice, Guinea pigs and dogs, the corneal endothelial cells are a mixture of hexagonal and pentagonal shaped cells in which their borders are irregular and interdigitating (Gibson, 1994; Smolin and Thoft, 1994; Krachmer et al., 1997; Nishida, 1997; Collin and Collin, 1998). However, Svaldeniene, et al. (2003) stated that the endothelial cells of the adult pig were square shaped; whereas in aging human they are not consistently hexagonal in shape, but rather pleomorphic in shape.

In $R$. rattus, the SEM showed that the endothelial cell surfaces were slightly bulging with many blebs, however in $M$. shawi, their surfaces were flattened and nearly smooth. Collin and Collin (1998) observed microvilli on many endothelial cells of human, rat, Gunia pig, dog and rabbit. However, a single primary cilium was a normal component of the rabbit endothelial cells (Gallagher, 1980) and was occasionally found in the endothelium of human (Beuerman and Pedroza,1996). Gordon et al. (1983) stated that, the endothelial surfaces in quiescent rat and rabbit were devoid of microvilli but display globular projections, however, the regenerating endothelial cells possess microvilli and filopodia. Yamaguchi et al. (1992) revealed several changes during the development of the rat corneal endothelial surfaces. These changes appeared as mushroom-like microvilli at 1 month-old, numerous filamentous microvilli at 3 months-old and numerous slender microvilli at 6 months-old.

Most probably, such controversies in the surface structures either microvilli, cilia, globular projections, blebs and filopodia in the rats and rabbits were related to the mitotic activity of the endothelial cells 
which are highly affected by their direct exposure to the surrounding aqueous humor of the eye. The TEM showed the presence of a small number but prominent large vacuoles, great numbers of vesiculated structures of the granular endoplasmic reticulum and mitochondria in the endothelial cells of $R$. rattus ; however, in $M$. shawi, highly granulated cytoplasm with some scattered vesiculated structures of the endoplasmic reticulum were observed. Such vesiculated structures of the endoplasmic reticulum were reported in many experimental mammals (Gibson, 1994; Nishida, 1997; Svaldeniene, et al., 2003). However, the presence of large vacuoles, highly vesiculated structures and mitochondria in $R$. rattus than those of $M$. shawi, most probably, is related to the high activity of these cells in the former species and their involvement in secretion, as well as active transport. Thus, it is not surprising that their primary function is to nourish and hydrate the cornea. This interpretation is highly logic in species inhabiting areas with continuous changeable environments as in $R$. rattus of the present investigation.

\section{References}

1. Amemiya, T. ; Yoshida, H.; Yoshida, M. and Kawaji, H. (1980). Ultrastructures of the normal surface of corneal epithelium of heterozygous rhino mouse with special reference to so-called epithelial holes. Albrecht Von Graefes Arch. Klin. Exp. Opthalmol., 213 (2): 101 - 107

2. Beuerman, R.W. and Pedrosa, L. (1996). Ultrastructure of the human cornea. Micros. Res. Tech., 33: $320-335$.

3. Chakravarti, S. (2001). Review. I . The cornea through the eyes of knockout mice. Exp. Eye Res., 73: $411-419$.

4. Collin, H.B. and Collin, S.P.(2000). A comparative SEM study of the vertebrate corneal epithelium.Cornea, $19: 218-230$.

5. Collin, S.P. and Collin, H.B.(1998). A comparative study of the corneal endothelium in vertebrates. Clin. Exp. Optom., 81 (6): 245 - 254.

6. Doughty , M. J. (1996). Evidence for heterogeneity in a small squamous cell type (light cells) in the rabbit corneal epithelium. A scanning electron microscope study. Corneal epithelial squamous. Doc. Opthalmol., 92 (2): 117 - 136.
7. Doughty, M. J. and Bergmanson, J.P. (2004). Collagen fibril characteristics at the corneo-scleral boundary and rabbit corneal stromal swelling. Clin. Exp. Optom., 87(2): $81-92$.

8. Doughty,M.J.;Seabert, W.; Bergmanson, J.P. and Blocker, Y. (2001). A descriptive and quantitative study of the keratocytes of the corneal stroma of albino rabbits using transmission electron microscopy. Tissue Cell, 33 (4): 408 - 422.

9. El-Dawi, E.F.A. (2002). A comparative study of the corneal structure of two marine teleosts Aphanius dispar and Gerres oyena . Egyp.J.Aquat.Biol. Fish., 6 (2): 207 - 235.

10. El-Dawi, E.F.A. (2004). Comparative studies on the structural adaptation of the cornea of the amphibious mudskipper fish, Periophthalmus waltoni and the maculated toad, Bufo regularis. Egyp. J. Aquat. Biol. Fish., 8 (2): 207 - 235.

11. Freud, D.E.; McCally, R.L.; Farrel, R.A. ; Cristol, S. L'hernault, N. and Edlhauser, H. (1995). Ultrastructure in the anterior and posterior stroma of perfused human and rabbit corneas. Relation to transparency. Invest. Opthalmol. Vis. Sci., 36: $1508-1523$.

12. Gallagher, B.C. (1980). Primary cilia of the corneal endothelium. Amer. J. Anat., $159: 475-484$

13. Gibson,I.K. (1994). Anatomy of the conjunctiva, cornea and limbus. In: The Cornea. Scientific Foundations and Clinical Practice. $3^{\text {rd }}$ ed., Smolin, G. and Thoft, R.A. (eds.), Little Brown and Company (Publ.,). Boston, New York, Toronto,London, Chapter, 1, pp 3-111.

14. Gordon, S.R.; Rothstein, H. and Harding, C.V. (1983). Studies on corneal endothelial growth and repair. IV. Changes in the surface during cell division as revealed by scanning electron microscopy. Eur. J. Cell Biol., 31 (1): 26 - 33.

15. Haustein, J. (1983). On the ultrastructure of the developing and adult mouse corneal stroma. Anat. Embryol., (Berl.), 168 (2): $291-305$.

16. Hayashi, S.; Osawa, T. and Tohyama, K. (2002). Comparative observation on corneas, with special reference to Bowman's layer and Descemet's membrane in mammals and amphibians. J. Morphology, 254(3): $247-258$.

17. Hodson, S.; Miller, F. and Riley, S. (1977). The electrogenic pump of rabbit corneal endothelium. Exp. Eye Res.,4 : 249-253.

18. Krachmer, J.H.; Mannis, M.J and Holland, E.J. (1997). Cornea. Fundam- 
entals of Cornea and External Disease. Vol., 1, Mosby-Year Book, Inc., St.Louis, New York, Sydney and Tokyo.

19. Lois, N.; Dawson, R.; McKinnon, A.D. and Forrester, J.V. (2003). A new model of posterior capsule opacification in rodents. Invest. Opthalmol. Vis. Sci., 44: 3450 - 3457.

20. Nishida, T. (1997). Basic Science: Cornea , Sclera and Ocular Adnexa Anatomy, Biochemistry, Physiology and Biomechanics. In : "Cornea. Fundamentals of Cornea and External Disease. Vol., 1, Krachmer, J.H.; Mannis, M.J and Holland, E.J. (eds), Mosby-Year Book, Inc. (Publ.), St.Louis, New York, Sydney and Tokyo. Section 1, Chapter 1, pp 3-28.

21. Sasaki, K.; Fukudome, H. and Inoue, T. (1999). Scanning electron microscopic studies of the basal surface of corneal endoth-elium and the stromal and endothelial surface of Descemet's membrane in rats. Nippon Ganka Gakkai Zasshi., 103 (2): 92- 98

22. Smolin, R.A. and Thoft, G.(1994). The Cornea. Scientific Foundations and Clinical
Practice. $3^{\text {rd }}$ ed., Little Brown and Company Publ., Boston, New York, Toronto,London.

23. Svaldeniene, E.; Babrauskiene, V. and Paunksniene, M. (2003). Structural features of the cornea : Light and electron microscopy. Veterin. JR Zootechnika., T. 24 (46): $50-55$.

24. Svedbergh, B. and Bill, A. (1972). Scanning electron microscopic studies of the corneal endothelium in man and monkeys. Acta Ophthalmol., 50: 321 336.

25. Takami, Y.; Gong, H. and Amemiya, T. (2004). Riboflavin deficiency induces ocular surface damage. Opthalmic Res., 36 (5): $299-302$.

26. Wassif, K. (1995). Guide to Mammals of Natural Protectorates in Egypt. Department of Natural Protectorates. Egyptian Environmental Affairs Agency (EEAA), Cabinet of Ministers, Cairo, A. R. Egypt

27. Yamaguchi, K.; Yamaguchi, K. and Turner, J. E. (1992). Scanning electron microscopic observation of the corneal endothelium in the Royal College of Surgeons rat. Nippon Ganka Gakkai Zasshi., 96 (1): 28 -33. 


\section{دراسات مقارنة للتكيف التركيبى للقرنية لإثنين من القوارض فى بيئات مختلفة}

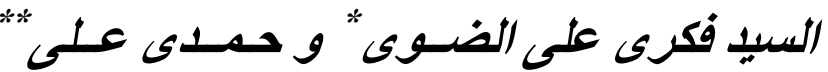

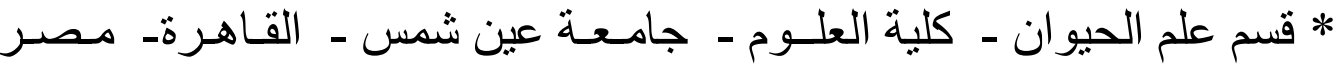

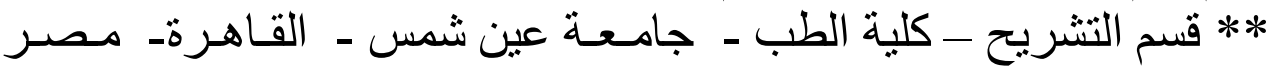

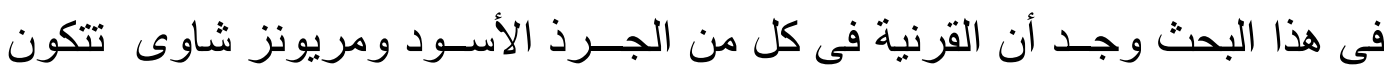

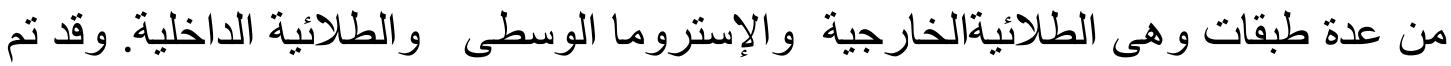
حساب متوسط سمك هذه الطبقات في كل منهما.

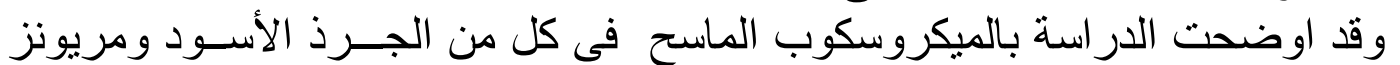

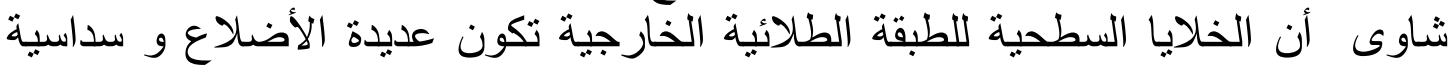

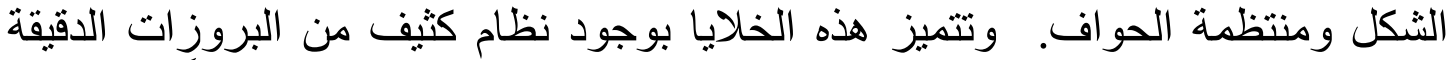

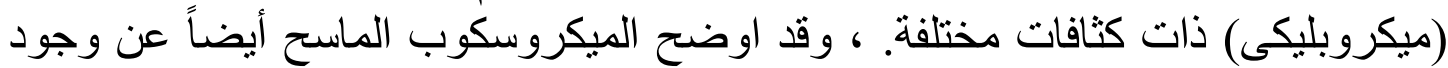

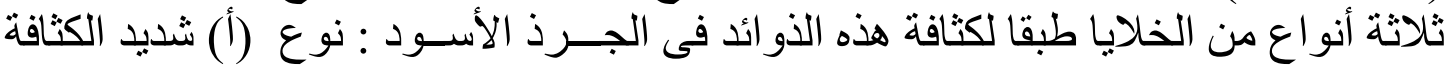

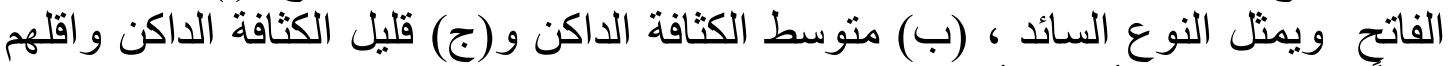

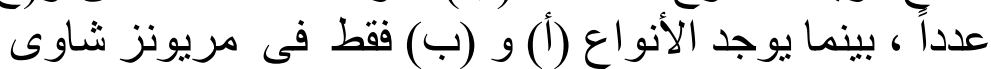

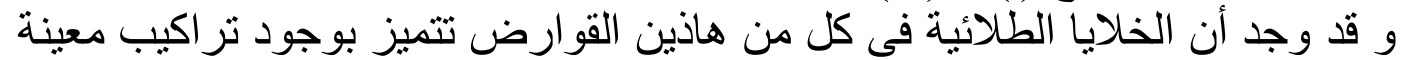

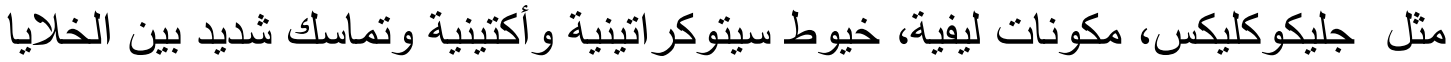

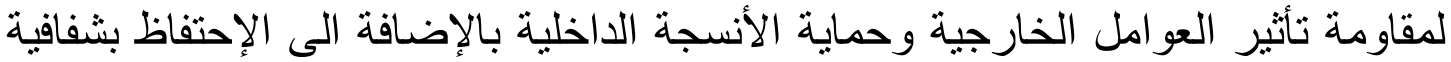

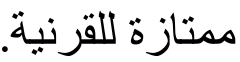

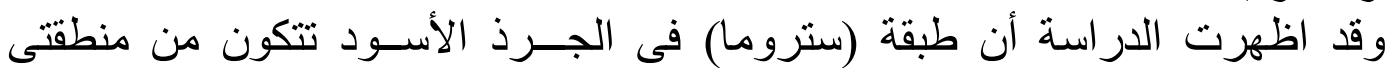

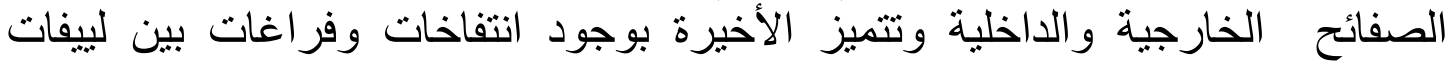

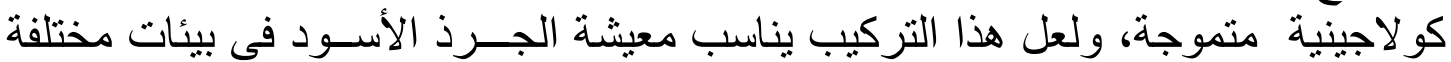

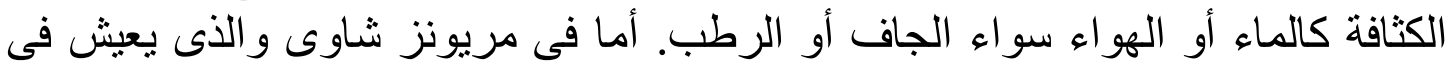

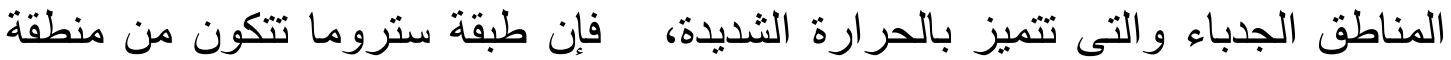

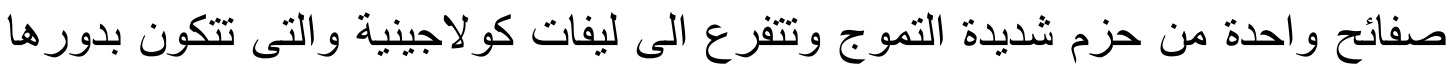

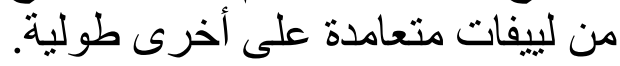

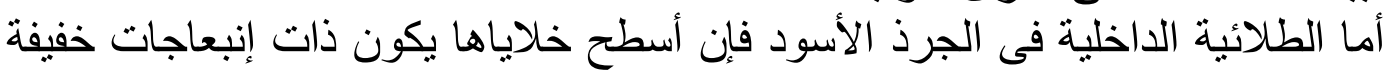

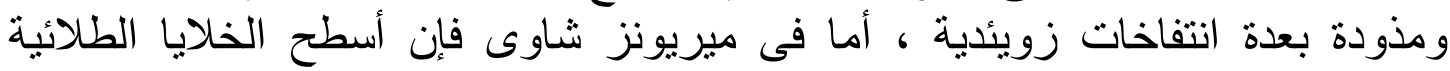

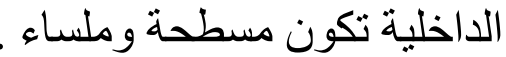
وقد أستنتج من هذا البحث أن ثركيب القرنية فى الجرذ الأسود تمكنه من المعيشة

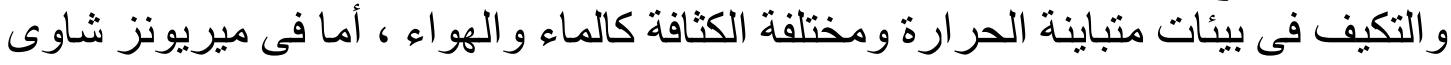
فإن تركيب القرنية تلائم الحياة فى الصحر اءو والتى تتميز بالجفاف وشدة الحرارة. 